Service social

\title{
Pour ajouter de la misère à la vie : L'impact d'une épidémiologie social-étatique sur l'action communautaire et les problèmes sociaux
}

\section{Michel Parazelli}

Volume 39, numéro 2, 1990

Les problèmes sociaux

URI : https://id.erudit.org/iderudit/706484ar

DOI : https://doi.org/10.7202/706484ar

Aller au sommaire du numéro

Éditeur(s)

École de service social de l'Université Laval

ISSN

1708-1734 (numérique)

Découvrir la revue

Citer cet article

Parazelli, M. (1990). Pour ajouter de la misère à la vie : L'impact d'une épidémiologie social-étatique sur l'action communautaire et les problèmes sociaux. Service social, 39(2), 175-187. https://doi.org/10.7202/706484ar

\section{Résumé de l'article}

Dans ce texte, l'auteur présente une réflexion critique sur la stratégie d'encadrement étatique des pratiques communautaires, dans une approche techno-médicale des problèmes sociaux et de ses conséquences sur l'analyse même des problèmes sociaux. Cette réflexion s'inscrit dans l'analyse de la réforme actuelle des services de santé et des services sociaux. Après avoir présenté les différents aspects de cette réforme, l'auteur fait ressortir l'opposition entre l'approche des problèmes sociaux soutenue par l'État et celle défendue par les organismes communautaires autonomes. Par ailleurs, l'auteur critique le projet de réforme en soulignant notamment ses effets néfastes pour la population en général et en particulier pour les jeunes. Il dénonce également l'utilisation en sous-traitance des groupes communautaires par l'État. 


\section{Pour ajouter de la misère à la vie L'impact d'une épidémiologie sociale-étatique sur l'action communautaire et les problèmes sociaux*}

Michel Parazelli

La présente réforme de la Loi sur les services de santé et les services sociaux interpelle à plus d'un point les organismes communautaires autonomes au Québec. Depuis plus de vingt ans, les intérêts dominants de décideurs politiques semblent plutôt privilégier le pouvoir techno-médical et l'approche commerciale du social et ce, malgré les avertissements répétés de nombreux groupes communautaires au sujet des conséquences sociales désastreuses qui découlent de ces orientations. Mais outre les effets sur la population, en particulier chez les jeunes, ce projet de réforme pourrait avoir des répercussions dangereuses sur la vie démocratique au Québec.

\section{Le projet de régionalisation : une décentralisation factice}

Contrairement au discours véhiculé par le ministère de la Santé et des Services sociaux (MSSS), le projet de régionalisation tel que formulé par l'avant-projet de loi est une fausse décentralisation politique. Cette opération de "régionalisation " demeure initiée, orientée, dirigée et évaluée par le pouvoir central. En fait, ce qui est proposé par le MSSS est davantage une décentralisation technique.

En effet, nous ne pouvons pas vraiment parler d'un réel partage de pouvoirs entre le MSSS et les régions lorsque celui-ci propose de ne décentraliser que les tâches d'organisation du travail à effectuer. De toute évidence, le ministère ne partage pas les pouvoirs liés à I'identification des paramètres d'intervention mais laisse tout au plus 
les régions sélectionner leurs paramètres parmi ceux qu'il a déjà définis. Cette attitude politique correspond davantage à une déconcentration de la conception du plan central dans les régions. Quant à la décentralisation, elle se résume à l'exécution des tâches régionales. II en est de même pour les objectifs et les programmes cadres (voir tableau 1). Le ministère décide des orientations à tous les niveaux de la production de services. Les régies régionales exécutent le travail à faire et ce, à l'intérieur des limites et du cadre de gestion déjà tracés par Québec. Dans ce contexte, le rôle d'une régie régionale se réduit à une agence de l'État.

Le ministère a même été jusqu'à définir les résultats escomptés (en \%) pour plusieurs paramètres d'intervention! II ne reste aux régions qu'à se transformer, au mieux de leur spécificité régionale, en décalques des volontés du pouvoir central en exécutant les tâches selon les devis du ministère.

Même le contexte structurel et les conditions politiques de la participation de la population à la gestion des services soulèvent de sérieuses questions au plan de la démocratie locale. En effet, on élimine ce qui reste de dynamismes locaux au profit de " monstres sous-régionaux " (la fusion des Centres d'accueil d'établissements) soumis eux-mêmes à une régie régionale. En ce sens, les fusions d'établissements constituent une dépossession des pouvoirs locaux.

\section{Un système centré sur les résultats statistiques}

On parle beaucoup d'efficacité et d'efficience, mais à quel sujet ? Pour le ministère, l'objectif du système est d'ajouter des années, de la santé et du bien-être à la vie. Il semble clair, pour les décideurs politiques, que l'efficacité des services administrés par le système se mesure en nombre de problèmes réduits, que ce soit au niveau de la santé ou des problèmes sociaux. En d'autres mots, il s'agit de comptabiliser des résultats de type quantitatif pour mesurer l'efficacité des services et des programmes.

Selon nous, l'efficacité et l'efficience mises de l'avant dans le projet de réforme relèvent plutôt de motivations électoralistes. En premier lieu, il s'agit de rendre visibles, de façon efficace, les actions posées par le gouvernement, c'est-à-dire de transformer de plus en plus les actes médicaux et les relations d'aide en objets statistiques. Pour ce faire, on va jusqu'à traiter les réalités sociales comme autant de problèmes à comptabiliser qu'il y a de spécialistes, sans se soucier des impacts négatifs de cette opération sur l'existence humaine.

$C^{\prime}$ est ce qui explique l'importance que les décideurs gouvernementaux accordent aux statistiques et aux chiffres qui permettent, par exemple, de justifier les dépenses lors d'une conférence de 


\section{TABLEAU 1}

\section{Une " décentralisation technique "}

Niveaux de partage des pouvoirs en fonction des champs de responsabilité

Avant-projet de loi sur la santé et les services sociaux

\begin{tabular}{|c|c|}
\hline Déconcentration & Décentralisation \\
\hline $\begin{array}{l}\text { - Les paramètres d'in- } \\
\text { tervention } \\
\text { - Les stratégies d'action } \\
\text { - La conception de pro- } \\
\text { grammes cadres } \\
\text { - Les modes de gestion } \\
\text { - Les objectifs } \\
\text { - Les résultats escomp- } \\
\text { tés } \\
\text { - Les modes d'allocation } \\
\text { des budgets } \\
\text { - Les modes d'organisa- } \\
\text { tion des pouvoirs } \\
\text { - Les modes d'évalua- } \\
\text { tion }\end{array}$ & $\begin{array}{l}\text { - Les études des besoins régionaux } \\
\text { - L'élaboration des programmes } \\
\text { - L'administration des programmes } \\
\text { - L'établissement des priorités } \\
\text { régionales } \\
\text { - La mise sur pied du collège élec- } \\
\text { toral } \\
\text { - La distribution des budgets } \\
\text { - La fusion des Centres d'accueil } \\
\text { (CA) d'établissements } \\
\text { - Le traitement des plaintes } \\
\text { - Les mécanismes de concertation } \\
\text { - La promotion de la participation } \\
\text { des citoyens } \\
\text { - Le travail d'évaluation }\end{array}$ \\
\hline
\end{tabular}

presse. Voilà pourquoi les ministères et les appareils d'État font appel à l'École des hautes études commerciales (HEC) ou à l'École nationale d'administration publique (ENAP) pour concevoir des systèmes de gestion, et fonctionnent au rendement, comme l'entreprise privée. Nous pouvons le constater dans le document d'orientations de l'ex-ministre Lavoie-Roux : la statistique devient le résultat escompté.

II s'agit, pour le ministère, de mettre en marché des produits tels que le "plan de services individualisés en santé mentale", le " traitement du sida ", la " campagne du non à la drogue " et, tout récemment, la "prévention de la violence ", en ciblant des consommateurs appelés pour l'occasion "des clientèles à risque ", afin de bien rendre visible (en \%) ce qui est produit par les services d'État. Dans ce contexte, orienter les objectifs sur les résultats n'est pas plus vertueux que de les orienter sur les services : cela revient au même.

\section{L'internalisation de l'action communautaire}

Depuis la naissance des groupes communautaires, aucun gouvernement n'est allé aussi loin dans l'encadrement étatique des pratiques 
communautaires. En effet, le MSSS propose carrément aux organismes communautaires de s'intégrer à la même structure de gestion administrative que les appareils d'État pour recevoir un support financier qui ne vise que les activités et interventions clairement identifiées au domaine de la santé et des services sociaux.

Jusqu'à maintenant, la majorité des actions communautaires touchant les services sociaux et de santé échappait à l'État et à ses appareils. De par la diversité de leurs approches, de leurs moyens et milieux d'intervention, les organismes communautaires constituent un bassin d'expérimentations sociales unique et représentent ainsi une richesse collective québécoise. Pour l'État, l'action communautaire produite par les organismes à but non lucratif est davantage perçue comme un moyen économique et disponible pour rentabiliser les interventions visant la réduction des problèmes sociaux et de santé. Cependant, un problème se pose. $\mathrm{D}^{\prime}$ une part, les organismes communautaires travaillent tous de façon différente sur une multitude de réalités sociales et culturelles; d'autre part, la plupart des organismes communautaires mettent davantage l'accent sur la prise en charge des personnes par elles-mêmes que sur la réduction proprement dite des problèmes sociaux. C'est ce qui explique les fortes résistances des organismes à servir les intérêts du système plutôt que ceux des citoyens et citoyennes avec qui ils travaillent.

La solution, pour le ministère, est donc d'internaliser les actions communautaires considérées par les gestionnaires comme des externalités devant éponger les débordements négatifs ou positifs produits par un système public déficient. Que ce soit suite à la déshumanisation de certains services sociaux ou à cause de l'absence de services publics pour répondre à de nouvelles réalités, ou encore par la mise sur pied de ressources alternatives par les appareils d'État euxmêmes, les organismes communautaires auraient jusqu'à maintenant toujours été considérés, par l'État, comme des ressources complémentaires au réseau public. Mais jamais encore avait-on envisagé une intégration aussi complète. En internalisant les actions communautaires à la structure administrative du réseau public, le MSSS oblige en quelque sorte les organismes communautaires à devenir les partenaires du système en échange d'un financement plus stable (étant donné leur situation de précarité financière).

Ces " nouveaux partenaires " constitueront un bassin important de ressources supplémentaires à utiliser pour désengorger le tropplein de clientèles dans le réseau. De plus, la standardisation des stratégies d'intervention et des modes d'évaluation, bref, de la production des services, s'effectuera aussi sur les actions des organismes communautaires, étant donné leur position minoritaire au sein des espaces de pouvoir. 
Effectivement, deux personnes issues du "secteur " communautaire, à côté de quinze individus en provenance d'autres secteurs, feront partie de la régie régionale. Au niveau purement mathématique et à partir de nos expériences précédentes, nous pouvons prévoir que les personnes issues du communautaire seront marginalisées à chaque fois qu'elles ne seront pas d'accord avec l'orientation dominante des établissements. II en résultera plus de services à moindres coûts, intégrés dans une hiérarchisation de la production standardisée. Le communautaire devient un maillon de plus dans la grande chaîne des bénéfices des services sociaux, un "bas d'écluse " du système pour gérer la crise des listes d'attente et des débordements de clientèles qui sévit actuellement dans le système.

Nous sommes donc contraints de revendiquer une reconnaissance de nos associations locales et de notre structure de représentation (regroupement), à l'extérieur de la structure de régionalisation.

\section{Une approche techno-médicale du social : l'épidémiologie sociale}

Comme nous l'avons mentionné plus haut, les organismes communautaires jeunesse puisent leurs orientations et leurs objectifs directement chez leurs membres, selon des processus démocratiques locaux. Étant donné que les orientations et les objectifs sont déjà définis par le ministère, il serait donc hasardeux pour les organismes communautaires de s'aventurer dans des sentiers qu'ils n'ont pas choisis et des directions qu'ils n'ont pas décidées. Par ailleurs, s'ils acceptaient de s'impliquer, ils se buteraient à une solide contradiction qui réside justement dans I'approche que le MSSS a développée et qu'il propose dans ses orientations pour aborder les problèmes sociaux. Pour le ministère, il s'agit de développer " une véritable épidémiologie sociale qui permettra de préciser les buts poursuivis en cours de route $»^{1}$ (Orientations, p. 19).

Voyons en quoi cette orientation est contradictoire. L'organisme communautaire qui voudrait recevoir un soutien financier pour travailler sur les causes d'un problème social donné devra se soumettre à une conception biomédicale du social pour être admissible à un programme quelconque. Cette conception médicale du social n'intervient, en fait, qu'au niveau du traitement des symptômes et non d'une démarche de socialisation et de prise en charge des citoyennes et citoyens par eux-mêmes. En effet, cette épidémiologie sociale ${ }^{2}$, telle que décrite dans les orientations du MSSS, consiste en une adaptation peu orthodoxe entre deux concepts issus de disciplines différentes : la biologie (médecine) et la 
sociologie. Au niveau méthodologique, cet emprunt (épidémiologie) à la biologie pour analyser le social constitue une limite sérieuse. Les rapports sociaux sont les produits d'une culture et non d'un corps organique, encore moins l'objet de pathologies. Enfin, au niveau pédagogique, l'exercice métaphorique est intéressant mais, pour élaborer une politique, cela manque de rigueur. Le danger dans l'usage d'une telle approche réside dans la tendance croissante à dissimuler les causes sociopolitiques des problèmes sociaux sous le couvert de pathologies sociales. Par exemple, même une cause de problèmes sociaux, telle la pauvreté, se transforme en une caractéristique pathogène, $c^{\prime}$ est-à-dire un symptôme d'une clientèle à risque, et n'est plus vue comme une des causes des multiples réactions sociales aux fortes pressions économiques. Les conséquences en sont dramatiques car les programmes ainsi élaborés viseront, à l'aide d'indices de prévalence et de déterminants environnementaux, à contenir la circulation de "virus sociaux" tels que la violence, la délinquance ou l'itinérance, au sein de clientèles à risque. Évidemment, cela aura pour effet d'augmenter la visibilité statistique de la "décontamination sociale " mais non de régler les problèmes à la source, c'est-à-dire l'absence de pouvoir de l'individu sur sa vie sociale et son habitat.

Cette normalisation médicale des problèmes sociaux aura comme conséquence de forcer les groupes communautaires à traiter les personnes comme étant elles-mêmes responsables de leurs problèmes, en les invitant à suivre une thérapie afin de modifier leur comportement à risque plutôt que d'acquérir un pouvoir sur les conditions sociales ambiantes.

La participation des organismes communautaires à l'élaboration des programmes régionaux est piégée à l'avance puisque, en plus, ces organismes seront en rapport de force minoritaire face aux établissements au sein des instances décisionnelles régionales.

\section{L'impact sur l'action communautaire}

Comme les organismes communautaires puisent leurs mandats et objectifs chez les citoyens et citoyennes, ils sont d'abord et avant tout redevables à leurs membres par le biais des assemblées générales et des conseils d'administration. Au-delà des diversités de moyens $d^{\prime}$ intervention et d'approche, les organismes communautaires ont, de façon générale, les mêmes objectifs de fond : la prise en charge des citoyennes et citoyens par eux-mêmes, la socialisation (volontaire) des rapports humains entre les citoyens et leur communauté, l'action sur les causes des problèmes sociaux et de santé. Donc, pour les 
organismes communautaires, répondre aux objectifs élaborés par leurs membres, c'est satisfaire les intérêts de ces derniers. C'est pourquoi plusieurs de ces organismes ont développé l'autogestion, la collégialité, la coopération ou des modes de travail caractérisés par une hiérarchisation très faible des rapports de production.

Comme leur rôle se verra réduit à celui de simples exécutants des mandats institutionnels des différents établissements, les organismes communautaires seront contraints de changer leur orientation d'action communautaire pour être supportés financièrement. Ils deviendront alors des sous-traitants de services sociaux et seront forcés d'adopter les finalités programmées par le ministère, qui ne prévoit pas le financement des activités inhérentes à la vie associative. En d'autres mots, le projet de régionalisation annonce l'institutionnalisation complète des organismes communautaires en décrétant le partenariat obligatoire entre organismes communautaires et établissements publics.

Pour les organismes en situation de survie, les conséquences qui en découlent sont les suivantes : souffrir d'amnésie volontaire quant au rôle de l'action communautaire autonome dans la société, pour obtenir un financement adéquat; ou bien devenir une ombre bénévole de l'action communautaire. En fait, nous risquons de perdre ce qu'il y a en amont de la notion de services, à savoir l'implication des citoyens et citoyennes dans un processus de prise en charge de leurs propres conditions d'existence. Nous appelons cela « la vie associative ", condition essentielle pour l'efficacité et l'efficience de l'action communautaire des organismes. Il s'agit là d'une nécessité pour briser l'isolement, créer des liens sociaux de façon volontaire et mettre des conditions en place pour que les personnes s'approprient un pouvoir sur leurs actes sociaux et deviennent critiques face aux enjeux collectifs de la vie en société.

En ne devenant que des sous-traitants du réseau public, les organismes communautaires perdront la souplesse d'action concernant les nouveaux problèmes et les nouvelles réalités, la proximité culturelle auprès des personnes dans leur milieu de vie, l'approche globale (autant individuelle que sociale), l'efficience effective de leur fonctionnement démocratique, la capacité de rejoindre les personnes exclues du réseau gouvernemental, et leur conscience sociale (en tant qu'actes de solidarité et de partage).

On coupe ainsi les derniers ponts qui existent entre la société, le gouvernement et les réalités globales des personnes marginalisées et exclues de la société québécoise.

II faut bien comprendre ici que l'enjeu se situe dans le fait que les citoyens et citoyennes ne pourront plus être supportés financièrement s'ils veulent s'associer entre eux et avec d'autres membres 
de la communauté pour d'autres raisons que celles autorisées par l'État. Par cette manière de gérer le social, l'État et ses appareils se mettront " hors jeu » de certains milieux de vie et sûrement de ceux des jeunes. Il est important de se rendre compte que la catastrophe produite par cette réforme affectera d'une manière insidieuse la qualité de la vie démocratique. Comment le gouvernement pourra-t-il orienter des politiques sociales s'il perd contact avec les réalités d'une partie de plus en plus importante de la population québécoise?

\section{L'impact sur les jeunes : une plus grande marginalisation}

Engagés dans certaines voies d'expérimentation sociale, les jeunes sont confrontés à des apprentissages décisifs pour leur insertion dans la société : l'initiation aux normes socioculturelles. Transgression et conformisme sont inhérents à cette expérimentation. Cela ne va pas sans heurt, surtout lorsque les espaces d'expérimentation se réduisent à des lieux marginaux. Jusqu'à maintenant, pour répondre à cette marginalisation croissante, l'État (par ses appareils : écoles, centres d'accueil, CSS, bureaux d'aide sociale, etc.) a maintenu le cap de la perspective économiste par des politiques d'exclusion, de dépossession et de coercition ${ }^{3}$, que ce soit dans le domaine du non-travail, du décrochage scolaire ou de l'adaptation sociale en général.

Pourtant, à cause de cette orientation gouvernementale, nous savons tous, depuis déjà trop longtemps, que les difficultés d'insertion sociale vécues par les jeunes ne font que s'accroître, qu'il s'agisse du chômage, de l'itinérance, du décrochage scolaire, de l'exclusion institutionnelle, etc. Selon toutes apparences, la technocratie d'État a conservé une orientation répressive au nom de la prévention des problèmes chez les jeunes. Nous faisons ici référence à la rééducation conformiste des centres d'accueil, aux projets éducatifs misant exclusivement sur l'excellence académique, aux campagnes " non à la drogue ", à la politique d'appauvrissement des jeunes liée à la réforme de l'aide sociale, etc. Bref, des politiques qui mettent en œuvre une "répression préventive " des comportements. Ces techniques pseudo-préventives préconisent les modifications de comportement comme remède magique. D'ailleurs, un certain courant de la criminologie enfonce le clou dans cette direction behavioriste en prétendant détecter les futurs délinquants dès la maternelle ! Est-ce que le MSSS classe ce type d'intervention dans la catégorie « prévention précoce "? 
Nous constatons que l'avant-projet de loi ainsi que les orientations liées à la réforme reconfirment et consolident la voie déjà empruntée par l'État, c'est-à-dire considérer les jeunes en tant que problèmes sociaux et définir leur existence de façon opérationnelle en les incluant, le cas échéant, dans une clientèle à risque faisant l'objet d'un programme d'intervention. Continuer dans cette direction, c'est renforcer un processus d'aliénation de la vie sociale des jeunes où la dépendance aux valeurs et aux directives morales des adultes prend le pas sur leur autonomie, même celle de penser!

Las de se faire considérer en objets, de plus en plus de jeunes " choisissent " de vivre en rupture avec le monde institutionnel parce que ce dernier est davantage préoccupé à justifier son travail qu'à répondre à leurs besoins. On peut presque qualifier cette situation "d'état de non-service" du monde institutionnel pour les jeunes.

Les jeunes ont davantage besoin d'espaces d'expérimentation où ils peuvent apprendre de façon collective, avec des adultes comme ressources, à devenir critiques face aux enjeux de la vie en société. Des lieux où ils peuvent être écoutés et supportés dans leur cheminement d'expérimentations sociales sans que leur itinéraire ne soit jonché uniquement d'interdits. Jusqu'à maintenant, les organismes communautaires jeunesse autonomes ont maintenu et maintiennent encore, "à bout de bras", ces espaces d'implication, de création et d'émancipation. Mais, comme nous l'avons vu précédemment, les organismes communautaires devront changer leur orientation pour être éligibles au support financier de la régie régionale. En réduisant ainsi les organismes communautaires jeunesse à des "thérapeutes de la pauvreté " ou de la violence (épidémiologie sociale oblige!), les jeunes devront traduire leurs problèmes et besoins de socialisation en "maladie " pour avoir accès à une ressource du milieu. On augmente alors la pression sous le couvercle de la marmite.

Une fois les organismes communautaires institutionnalisés, nous sommes convaincus qu'une bonne partie des jeunes déserteront ce type de ressources. Comme s'il ne devait leur rester que la rue, la violence, les gangs, la prostitution et le suicide pour se socialiser ! Alors les problèmes sociaux ne feront que s'accroître.

\section{Une consolidation de la gestion permanente du provisoire}

Les conséquences de cette gigantesque opération mécanique de rentabilité politique ne seraient pas catastrophiques s'il s'agissait d'une production matérielle d'objets de consommation comme à la 
Régie des alcools, par exemple. Le problème vient du fait qu'il s'agit de vies humaines, de solidarités sociales, de supports affectifs, de valeurs socioculturelles, bref de subjectivité et de conscience sociale dans sa plus grande production de sens. Comme société, nous nous engageons dans la gestion permanente (en tout cas, d'une façon institutionnalisée) de solutions provisoires, en ce sens que les problèmes sociaux ne sont jamais élucidés dans une perspective causale des phénomènes sociaux. Ils sont plutôt entreposés dans des établissements ou relégués dans des projets d'entraide spécialisés (ex. : maintien à domicile) pour y être traités, éventuellement. Toutefois, les traitements se réduisent à des modifications de comportement, un peu à la manière d'antibiotiques ou de cures de désintoxication. En fait, lorsque le MSSS propose de fixer des objectifs visant la réduction des problèmes, il pousse ses entreprises publiques et les organismes communautaires à effacer plus efficacement les symptômes et à corriger les "erreurs " de comportement. Par une telle politique, nous pensons que le MSSS est en train de consolider ce que nous appelons "I'hypermarché des services sociaux", car cette "réforme " propose de maintenir le système public actuel dans un marché qui s'est lui-même excédé.

Pour les citoyens et citoyennes, il n'est même plus question de consommer des services. En effet, les " clientèles à risque " que sont devenus certains groupes ciblés par l'État, n'auront plus à chercher de réponse à leurs besoins, mais devront plutôt répondre à la question de fonctionnalité des objets construits par l'État en tant que problèmes sociaux. La réponse est incluse dans la question. En $\mathrm{d}^{\prime}$ autres mots, pour avoir accès à une ressource sociale, les citoyens et citoyennes devront s'intégrer dans une des " cases " autorisées par I'État. En se conformant aux exigences du système, ils domestiqueront leurs comportements sociaux pour s'adapter à la gestion technocratique de la représentation des fonctions sociales érigées en système.

\section{Le développement de la communautique}

En " arrimant " les organismes communautaires aux priorités standardisées des institutions, le système étatique est en voie de produire le modèle de toute forme future de socialisation contrôlée. L'État retranscrit donc tous les flux sociaux contradictoires en circuits intégrés. D'ailleurs, ce n'est que depuis peu d'années que les expressions "interface " et " arrimage " sont utilisées pour nommer la nature de la collaboration forcée entre les organismes communautaires et les établissements d'État. Même si certaines expériences semblent constituer des " modèles de réussite", nous pensons que 
le partenariat est plutôt devenu un "pattern-ariat", enfin une simulation de la collaboration communautaire à la construction des problèmes sociaux.

De cette manière, le système focalise en un espace-temps homogène toutes les fonctions dispersées du corps et de la vie sociale (travail, loisirs, relations, santé, culture, hébergement, etc.). Certains centres d'accueil pour jeunes systématisent cette déstructuration systémique du social à l'aide du "modèle satellite». Par leur fondation privée, ces centres d'accueil se dotent d'un système de ressources sans but lucratif, appelées "communautaires" pour l'occasion. Ces ressources dépendent toutes du contrôle d'une ou de plusieurs institutions et servent d'appendices de services à ces dernières. C'est ce que nous avons appelé la "communautique", une conception technocratique (écosystémique) de l'action communautaire où une fonction est hiérarchisée dans un système hyperspécialisé 4 . À titre d'exemple, un centre d'accueil de réadaptation pour jeunes a mis sur pied un centre d'hébergement pour ex-bénéficiaires qui n'ont pas réussi leur réinsertion dans la société. Alors, on recommence la rééducation avec une communauté et des moyens hyperspécialisés. On n'agit pas sur les causes sociales et institutionnelles de ces échecs; on gère les déracinements par une fausse pratique "communautaire", de telle sorte que la pratique communautique n'offre que des services spécialisés à une « communauté automatique " pour pallier aux carences institutionnelles.

En ce sens, la réforme de l'aide sociale devient une autre illustration de cette dynamique où, autant les travailleurs d'État que les "bénéficiaires " et les "prestataires" se retrouvent dépossédés par une machine qui leur fait violence quotidiennement. Être sans travail, aujourd'hui, c'est être suspect, donc représenter un problème. Cette réforme de l'aide sociale a alors prévu une batterie de spécialistes pour traiter les problèmes individuels qui nuisent au potentiel d'employabilité de chaque personne. De cette façon, les deux systèmes, "aide sociale " et "services sociaux et de santé ", seront liés par l'interface du traitement de la pauvreté, opération comparable aux célèbres fusions d'entreprises que nous avons connues ces dernières années.

Pour l'État, ce qui semble important, c'est de créer et de contrôler un immense mouvement de va-et-vient qui centralise et redistribue des populations à l'image des mouvements de circulation urbaine, ce qui a pour effet de disperser la capacité des gens à se socialiser en rassemblant de façon fonctionnelle le traitement des symptômes de leurs problèmes. Ce mouvement de rassemblement s'accompagne d'une évacuation vers d'autres lieux de rassemblement fonctionnels, et ainsi de suite. Jusqu'à ce que l'effet de ce brassage automatisé en 
exaspère plusieurs, au point de choisir alors l'isolement et la marginalité sociale. Et encore, peut-on vraiment parler de marginalité dans une société duale?

\section{Une morale de l'opérationnalité}

Ce travail d'acculturation institutionnelle procède selon « une morale de l'opérationnalité ». La finalité d'une fonction sociale donnée n'a même plus de valeur, sinon dans les discours politiques de persuasion en tant qu'argument de dissuasion. En d'autres mots, il n'est même plus important, pour les gestionnaires d'établissements, de réellement réinsérer un jeune dans la société. Ce qui importe, $c^{\prime}$ est que les statistiques de traitement et de réduction de problèmes se compilent bien, et avec efficacité.

Ici nous ne parlons pas de complot, ni d'effets de système, mais bien d'une complaisance socioculturelle aux valeurs nordaméricaines de la réussite économique subordonnées à une morale de l'opérationnalité. Les effets de système se situent toujours en aval du choix ou du non-choix politique.

\section{Vers la dualisation sociale}

Par ailleurs, cette gestion continuelle de solutions provisoires s'accompagne d'une privatisation idéologique de la vie en société, une recette parfaite pour renforcer la dualisation de la société et briser ce qui reste de conscience sociale au Québec. On peut logiquement s'attendre à ce que de telles conditions favorisent l'émergence de nouvelles classes dangereuses. Nouvelles manifestations culturelles de la pauvreté qui développent des réactions sociales multiformes et imprévisibles face aux pressions socio-économiques combinées à l'absence de repères d'insertion socioculturelle.

$\mathrm{Si}$ nous ajoutons à ce sombre portrait les conséquences des réformes auxquelles les groupes communautaires ont été confrontés cette année (entre autres l'aide sociale, l'assurance-chômage, la politique familiale, les politiques d'habitation) de même que les réformes qui auront des conséquences à long terme (le libreéchange, la TPS) et celles qui se préparent (les modifications à la Loi sur les jeunes contrevenants, le projet de loi sur l'avortement), nous pensons qu'à l'instar de certains pays occidentaux, le Québec consolide et élargit l'état de son sous-développement social, culturel et économique à un nombre de plus en plus important de ses citoyens et citoyennes. 


\section{Notes}

* Cet article est tiré du mémoire intitulé " Pour ajouter de la misère à la vie » présenté par le Regroupement des organismes communautaires jeunesse du Montréal métropolitain (ROCJMM) à la Commission parlementaire qui étudie l'avant-projet de loi sur les services de santé et les services sociaux du Québec. Le ROCJMM existe depuis 1976. Ses organismes membres travaillent sur de multiples réalités sociales en invitant les jeunes à identifier eux-mêmes leurs problèmes, leurs besoins et leurs projets par une association volontaire à l'organisme.

${ }^{1}$ Ministère de la Santé et des Services sociaux, Pour améliorer la santé et le bien-être au Québec : Orientations, Québec, 1989 : 19.

2 Épidémiologie : Étude des rapports existant entre les maladies et divers facteurs (mode de vie, milieu ambiant ou social, particularités individuelles) susceptibles d'exercer une influence sur leur fréquence, leur distribution, leur évolution. (Petit Robert 1, 1983 : 671).

Épidémie : du latin médiéval epidemia emprunté au grec epidêmos, "qui circule dans le peuple ". (Dictionnaire étymologique Larousse, 1971 : 270).

${ }^{3}$ Voir "Les réalités des jeunes : banalisation et coercition", document de réflexion présenté au Conseil Permanent de la Jeunesse, ROCJMM, avril 1989.

${ }^{4}$ Pour en savoir plus long, voir le texte "Si vous avez attrapé des tiques..." , document d'analyse sur les enjeux actuels et à venir de l'action communautaire, ROCJMM, Michel Parazelli, novembre 1987. 